Tropical Journal of Pharmaceutical Research October 2021; 20 (10): 2227-2233

ISSN: $1596-5996$ (print); 1596-9827 (electronic) (C) Pharmacotherapy Group, Faculty of Pharmacy, University of Benin, Benin City, 300001 Nigeria.

\title{
Effect of combination of Yiqi Jianpi Yangxue Decoction and chemotherapy on quality of life and adverse reactions of patients with recurrent ovarian cancer
}

\author{
Qingling Zhang ${ }^{1}$, Wenjuan Zhang ${ }^{2}$, Xiao Wang ${ }^{3}$, Xueling Chen ${ }^{2}$, Yewu Gao ${ }^{4 *}$ \\ ${ }^{1}$ Department of Traditional Chinese Medicine, ${ }^{2}$ Department of Obstetrics, The Second Children \&Women's Healthcare of Jinan \\ City, Jinan 271100, ${ }^{3}$ Department of Respiration, Tai'an Cancer Prevention and Treatment Hospital, Taian 271000, ${ }^{4}$ Department \\ of Gynecology, Zhangqiu District People's Hospital, Jinan 250200, Shandong Province, China
}

*For correspondence: Email: baohejiv@163.com

Sent for review: 10 May 2021

Revised accepted: 29 September 2021

\begin{abstract}
Purpose: To investigate the effect of combined treatment with Yiqi Jianpi Yangxue Decoction and chemotherapy (carboplatin + paclitaxel) on quality of life (QOL) and adverse reactions of patients with recurrent ovarian cancer (ROC).

Methods: One hundred and fourteen (114) ROC patients in The Second Children \& Women's Healthcare of Jinan City were split into chemotherapy group $(n=60)$ and combination group $(n=54)$, based on whether or not they were treated with Yiqi Jianpi Yangxue Decoction. Differences in clinical efficacy, adverse reactions, levels of tumor marker, levels of immune indexes, and scores on Karnofsky Performance Status (KPS) between the two groups were evaluated.

Results: Treatment effectiveness was higher in the combination group than in the chemotherapy group $(p<0.05)$. Compared with the chemotherapy group, post-treatment levels of HE4 and CA125 in the combination group were lower, while the levels of $\mathrm{CD}_{3}{ }^{+}, \mathrm{CD}_{4}{ }^{+}$and $\mathrm{CD}_{8}{ }^{+}$, and population of NK cells were higher $(p<0.05)$. After treatment, the KPS score in the combination group was higher than the corresponding score in the chemotherapy group $(p<0.05)$.

Conclusion: Combination of Yiqi Jianpi Yangxue Decoction and chemotherapy (carboplatin + paclitaxel) produces significant enhancement of clinical efficacy in the treatment of ROC. The combination treatment is highly safe, and improves the health status and QOL of patients. Therefore, the combination treatment appears to be suitable for the management of ovarian cancer.
\end{abstract}

Keywords: Recurrent ovarian cancer, Yiqi Jianpi Yangxue Decoction, Chemotherapy, Carboplatin, Paclitaxel, Adverse reactions, Quality of life

This is an Open Access article that uses a funding model which does not charge readers or their institutions for access and distributed under the terms of the Creative Commons Attribution License (http://creativecommons.org/licenses/by/4.0) and the Budapest Open Access Initiative (http://www.budapestopenaccessinitiative.org/read), which permit unrestricted use, distribution, and reproduction in any medium, provided the original work is properly credited.

Tropical Journal of Pharmaceutical Research is indexed by Science Citation Index (SciSearch), Scopus, International Pharmaceutical Abstract, Chemical Abstracts, Embase, Index Copernicus, EBSCO, African Index Medicus, JournalSeek, Journal Citation Reports/Science Edition, Directory of Open Access Journals (DOAJ), African Journal Online, Bioline International, Open-J-Gate and Pharmacy Abstracts

\section{INTRODUCTION}

Ovarian cancer (OC), a malignant tumor in the female reproductive organs, may occur at any age, but it is seen mostly in women over 50 years old [1-3]. Clinical medical statistics indicate that the incidence of $\mathrm{OC}$ ranks third, while its mortality ranks first among gynecological malignancies in China [4]. The early symptoms of OC are not easily noticeable, and the symptoms of advanced lesions are not typical. The major therapeutic strategies often used in clinical 
practice for OC are surgery, chemotherapy and targeted-drug therapy. However, these treatment methods often result in high levels of posttreatment recurrence. Moreover, recurrence during treatment is an important cause of death in OC patients [5-8].

Yiqi Jianpi Yangxue Decoction is a newly developed type of traditional Chinese medicine (TCM) which exerts an adjuvant effect on middle and advanced forms of ovarian cancer [9]. Nowadays, limited reports have been observed on the treatment of recurrent ovarian cancer (ROC) using combination of Yiqi Jianpi Yangxue Decoction and chemotherapy. Therefore, this paper was to explore the clinical effect of combined use of Yiqi Jianpi Yangxue Decoction and chemotherapy in the treatment of ROC. Moreover, the effects of this therapy on quality of life (QOL) and adverse reactions were determined, with a view to providing a new research direction for improved clinical treatment of ROC.

\section{METHODS}

\section{Patients}

One hundred and fourteen (114) ROC patients admitted in The Second Children \& Women's Healthcare of Jinan City (January 2019-January 2021) were chosen, and split into chemotherapy group $(n=60)$ and combination group $(n=54)$, based on whether or not they were given Yiqi Jianpi Yangxue Decoction. The study was approved by the institutional ethics committee, and conducted in accordance with the Declaration of Helsinki as revised in 2013 [10].

\section{Inclusion criteria}

Patients in the following categories were included in the study: those who were confirmed to have ovarian cancer after postoperative pathological examination, patients who were stable after undergoing six cycles of chemotherapy, those with increased serum levels of CA125, patients with lesions that were measurable with computed tomography (CT), ultrasound and magnetic resonance imaging (MRI) after chemotherapy, and patients with Eastern Cooperative Oncology Group (ECOG) score less than 2 points.

\section{Exclusion criteria}

Patients whose conditions were complicated with other malignant tumors or organic diseases, patients with expected survival times less than three months, and those who had communication or cognitive impairment, were excluded from the study. Moreover, patients who had incomplete clinical data were excluded.

This study obtained the approval of the Ethics Committee of The Second Children \& Women's Healthcare of Jinan City (approval no. 20181119). The purpose and procedures involved in the study were explained to the patients and their families, and they signed informed consent forms to indicate their willingness to participate in the study.

\section{Treatments}

Patients in the chemotherapy group were given paclitaxel (Yunnan Handle Bio-Tech Co. Ltd, specification: $30 \mathrm{mg}$; SFDA approval no. $\mathrm{H} 10960322)$ at a dose range of $135-175 \mathrm{mg} / \mathrm{m}^{2}$ solubilized in $500 \mathrm{~mL}$ of $0.9 \% \mathrm{NaCl}$ and administered via intravenous drip for no less than 3 h. In addition, the patients were given carboplatin (Qilu Pharmaceutical Co. Ltd.; SFDA approval no. H20020181; specification: $50 \mathrm{mg}$ ) at a dose of $350 \mathrm{mg} / \mathrm{m}^{2}$ or AUC of 5.0 - 7.5 $\mathrm{mg} / \mathrm{mL} / \mathrm{min}$, dissolved in $250 \mathrm{~mL}$ of $5 \%$ glucose solution and administered via intravenous drip for $1 \mathrm{~h}$, once every 3 weeks. The patients received 6 cycles of chemotherapy. During chemotherapy, the patients' symptoms were closely monitored. Before chemotherapy, the patients received 20 $\mathrm{mg}$ of dexamethasone (specification: $5 \mathrm{mg}$; manufacturer: Shanghai Xiandai Hasen Pharmaceutical Co. Ltd; SFDA approval no. H41021924) via intravenous injection; $30 \mathrm{mg}$ of cimetidine (specification: $0.2 \mathrm{~g}$; manufacturer: Guangzhou Baiyunshan Mingxing Pharmaceutical Co. Ltd.; SFDA approval No: $\mathrm{H} 44024199)$ via intramuscular injection, and 40 mg of diphenhydramine (specification: $20 \mathrm{mg}$; manufacturer: Beijing Yookon Pharmaceutical Co. Ltd; SFDA approval no. H11020523) via intramuscular injection, in order to prevent adverse reactions [11]. Patients in the combination group were given Yiqi Jianpi Yangxue Decoction obtained from $30 \mathrm{~g}$ of Astragalus membranaceus, $20 \mathrm{~g}$ of poria cocos, $15 \mathrm{~g}$ of oldenlandia, $10 \mathrm{~g}$ of curcuma zedoary, 10 $\mathrm{g}$ of dangshen, $10 \mathrm{~g}$ of kuh-seng, $10 \mathrm{~g}$ of ginger, $10 \mathrm{~g}$ of bighead atractylodes rhizome, $10 \mathrm{~g}$ of Paeonia lactiflora, $8 \mathrm{~g}$ of rhizoma sparganii and 8 $\mathrm{g}$ of monkshood. This resulted in $400 \mathrm{~mL}$ of Yiqi Jianpi Yangxue Decoction which was administered every morning and evening. Chemotherapy treatment was same as that in the chemotherapy group.

\section{Evaluation of treatment effectiveness}

Treatment effectiveness was categorized into three, based on CA125 level: complete response 
$(\mathrm{CR})$, partial response (PR), stable disease (SD) and progressive disease (PD). Patients were said to have CR if CA125 level was less than 35 $\mathrm{U} / \mathrm{mL}$, while PR was used for patients whose CA125 levels decreased by more than $50 \%$ for more than 4 weeks. Stable disease (SD) was used for patients whose CA125 levels decreased by 25 - $50 \%$ for more than 4 weeks, while PD was used for patients whose CA125 levels decreased by less than $25 \%$, or whose CA125 levels were increased. Total treatment effectiveness (TTE) was calculated as shown in Eq. 1:

TTE (\%) $=[($ No. of $C R+$ No. of PR $) /$ Total number of patients] $\times 100 \ldots . .(1)$

\section{Adverse reactions}

Adverse reactions were categorized based on the classification of adverse reactions in American Adverse Reaction Evaluation System for Generic Chemotherapy Drugs (Fourth Edition) [12]. Mild adverse reactions without symptoms or treatment, in addition to white blood cell (WBC) count of 3.0-3.9 $\times 10^{9} / \mathrm{L}$, hemoglobin (Hb) level of $95-100 \mathrm{~g} / \mathrm{L}$, and platelet (PLT) count of $75-99 \times 10^{9 /} \mathrm{L}$, were classified as level 1 . Moderate adverse reactions, with patients having WBC count of 2.0-2.9 $\times 10^{9} / \mathrm{L}$, Hb level of $80-94$ $\mathrm{g} / \mathrm{L}$, and PLT count of $50-74 \times 10^{9} / \mathrm{L}$ (without effect on life) were classified as level 2. Severe adverse reactions requiring active intervention in patients with WBC count of $1.0-1.9 \times 10^{9} / \mathrm{L}, \mathrm{Hb}$ level of 65-79 $\mathrm{g} / \mathrm{L}$, and PLT count of 25-49 $\times 10^{9} / \mathrm{L}$, were categorized as level 3 . Adverse reactions which caused organ failure and disabled or threatened life, with WBC count of < $1.0 \times 10^{9} / \mathrm{L}, \mathrm{Hb}<65 \mathrm{~g} / \mathrm{L}$, and PLT count < $25 \times 10^{9 /} \mathrm{L}$, were classified as level 4 . Adverse reactions that led to death were classified as level 5.

\section{Serum tumor markers}

Fasting peripheral venous blood samples were obtained from patients in the morning, before and after treatment, and serum samples were obtained after centrifugation. Chemiluminescence immunoassay was used to determine the contents of serum human epididymis secretory protein 4 (HE4) and carbohydrate antigen 125 (CA125). In these assays, HE4 > $150 \mathrm{pmol} / \mathrm{L}$ or CA125 > $35 \mathrm{U} / \mathrm{mL}$ indicated that the patients were positive.

\section{Immune function}

The $\mathrm{T}$ cell markers i.e., $\mathrm{T}$ cell subsets such as $\mathrm{CD}_{3}{ }^{+}, \mathrm{CD}_{4}{ }^{+}, \mathrm{CD}_{8}{ }^{+}$and $\mathrm{NK}$ cells, and their proportions, were determined directly or indirectly using immunofluorescence.

\section{Karnofsky Performance Status (KPS) scores}

The KPS score of patients were determined before and after treatment. The maximum score was 100 points. A higher score indicated that patients had better health status and could tolerate side effects caused by the treatment. Scores above $50-70$ points were classified as non-dependency, suggesting that the patients could take care of themselves. Scores of 50-70 points indicated semi-dependency which implied that the patients could basically take care of themselves. Scores below 50 points were indicative of dependency, meaning that the patients needed much help from others. Patients with scores above 80 points had good postoperative status and long survival time.

\section{Statistical analysis}

In this study, SPSS20.0 was used as data processing software, while GraphPad Prism 7 (GraphPad Software, San Diego, USA) was used for graphics. Count data and measurement data obtained in this study were subjected to statistical analyses using $x^{2}, t$-test and normality test. Differences were considered statistically significant at $p<0.05$.

\section{RESULTS}

\section{Comparison of general data on patients}

No marked differences in general patient profiles were observed between the two groups $(p>$ 0.05; Table 1).

\section{Clinical efficacy}

There was markedly higher total treatment effectiveness in the combination group than in the chemotherapy group $(p<0.05$, Table 2$)$.

\section{Incidents of adverse reactions}

Table 3 shows no statistic differences in incidence of adverse reactions between the two groups $(p>0.05)$.

\section{Serum levels of tumor markers}

In the chemotherapy group, the levels of HE4 before and after treatment were $274.35 \pm 51.32$ and $59.45 \pm 9.06$, respectively, while the corresponding levels in the combination group were 275.34 \pm 50.82 and $25.36 \pm 8.72$, respectively.

Trop J Pharm Res, October 2021; 20(10): 2229 
Table 1: Patients' profile

\begin{tabular}{|c|c|c|c|c|}
\hline Index & $\begin{array}{l}\text { Chemotherapy group } \\
(\mathrm{n}=60)\end{array}$ & $\begin{array}{l}\text { Combination } \\
\text { group }(n=54)\end{array}$ & $t / x^{2}$ & $P$-value \\
\hline Age (years) & $62.54 \pm 4.17$ & $61.86 \pm 3.97$ & 0.8893 & 0.3758 \\
\hline FIGO staging & & & 0.1303 & 0.718 \\
\hline I-II & $8(13.33 \%)$ & $6(11.11 \%)$ & & \\
\hline III-IV & $52(86.67 \%)$ & $48(88.89 \%)$ & & \\
\hline ECOG score & & & 0.0193 & 0.890 \\
\hline 0 & $33(55 \%)$ & $29(53.70 \%)$ & & \\
\hline $1-2$ & $27(45 \%)$ & $25(46.30 \%)$ & & \\
\hline Pathological types & & & 0.0100 & 0.920 \\
\hline Serous carcinoma & $35(58.33 \%)$ & $31(57.41 \%)$ & & \\
\hline Mucinous carcinoma & $19(31.67 \%)$ & $19(35.19 \%)$ & & \\
\hline Clear cell carcinoma & $6(10 \%)$ & $4(7.41 \%)$ & & \\
\hline $\begin{array}{l}\text { Withdrawal time of } \\
\text { chemotherapy (months) }\end{array}$ & & & 0.0199 & 0.888 \\
\hline$<3$ months & $14(23.33 \%)$ & $12(22.22 \%)$ & & \\
\hline$\geq 3$ months & $46(76.67 \%)$ & $42(77.78 \%)$ & & \\
\hline CA125 (U/mL) & & & 0.0287 & 0.865 \\
\hline$<100$ & $13(21.67 \%)$ & $11(20.37 \%)$ & & \\
\hline$\geq 100$ & $47(78.33 \%)$ & $43(79.63 \%)$ & & \\
\hline Seroperitoneum (mL) & & & 0.0293 & 0.864 \\
\hline$<200$ & $18(30 \%)$ & $17(31.48 \%)$ & & \\
\hline$\geq 200$ & $42(70 \%)$ & $37(68.52 \%)$ & & \\
\hline
\end{tabular}

Table 2: Comparison of clinical efficacy [n (\%)]

\begin{tabular}{|c|c|c|c|c|}
\hline Efficacy & Chemotherapy group $(n=60)$ & Combination group $(n=54)$ & $x^{2}$ & $P$-value \\
\hline CR & $5(8.33)$ & $11(20.37)$ & & \\
\hline PR & $9(15)$ & $14(25.93)$ & & \\
\hline SD & $26(43.33)$ & $19(35.19)$ & & \\
\hline PD & $20(33.33)$ & $10(18.52)$ & & \\
\hline TTE & $14(23.33)$ & $25(46.30)$ & 6.6586 & 0.010 \\
\hline
\end{tabular}

Table 3: Comparison of incidence of adverse reactions [n (\%)]

\begin{tabular}{lccc}
\hline Adverse reaction & Level & Chemotherapy group $(\mathrm{n}=60)$ & Combination group $(\mathrm{n}=54)$ \\
\hline Decrease in WBC & Levels 1-2 & $52(86.67)$ & $47(87.04)$ \\
& Level 3 & $7(11.67)$ & $5(9.26)$ \\
& Level 4 & $1(1.67)$ & $2(3.70)$ \\
Decrease in PLT & Levels 1-2 & $60(100)$ & $54(100)$ \\
Decrease in Hb & Levels 1-2 & $57(95)$ & $51(94.44)$ \\
& Level 3 & $2(3.33)$ & $2(3.70)$ \\
Melena & Level 4 & $1(1.67)$ & $1(1.85)$ \\
& Levels 1-2 & $58(96.67)$ & $54(100)$ \\
Nausea and vomiting & Level 3 & $2(33.33)$ & $0(0)$ \\
& Levels 1-2 & $60(100)$ & $53(98.15)$ \\
\hline
\end{tabular}

In the chemotherapy group, the CA125 levels before and after treatment were $202.14 \pm 52.85$ and $38.24 \pm 5.16$, respectively, while the corresponding levels in the combination group were $203.05 \pm 51.63$ and $16.25 \pm 5.43$, respectively. After treatment, the HE4 and CA125 levels in both groups were reduced, with lower HE4 and CA125 levels in the combination group. See Figures 1-2.

\section{KPS scores}

In the chemotherapy group, the KPS scores before and after treatment were $63.18 \pm 13.48$ and $71.55 \pm 12.67$, respectively, while the corresponding scores in the combination group were $62.59 \pm 12.35$ and $78.81 \pm 13.56$, respectively. After treatment, KPS scores in both groups were increased, with higher KPS score in the combination group. See Figure 3.

\section{Immune function indexes}

Table 4 shows notably higher levels of $\mathrm{CD}_{3}{ }^{+}$, $\mathrm{CD}_{4}{ }^{+}, \mathrm{CD}_{8}{ }^{+}$and $\mathrm{NK}$ cells in the combination group than in the chemotherapy group $(p<0.05)$.

Trop J Pharm Res, October 2021; 20(10): 2230 
Table 4: Comparison of levels of immune function indexes

\begin{tabular}{lcccc}
\hline Group & $\mathbf{C D}_{3}{ }^{+}$ & $\mathbf{C D}_{4}{ }^{+}$ & $\mathbf{C D}_{8}{ }^{+}$ & NK cells \\
\hline Chemotherapy $(\mathrm{n}=60)$ & $38.15 \pm 9.38$ & $19.34 \pm 4.55$ & $12.53 \pm 5.21$ & $10.32 \pm 3.22$ \\
Combination $(\mathrm{n}=54)$ & $55.24 \pm 7.81$ & $30.27 \pm 4.15$ & $20.33 \pm 5.14$ & $17.69 \pm 4.53$ \\
$X^{2}$ & 10.5055 & 13.3484 & 8.0322 & 10.0869 \\
$P$ & 0.000 & 0.000 & 0.000 & 0.000 \\
\hline
\end{tabular}

Values are presented as mean \pm SD

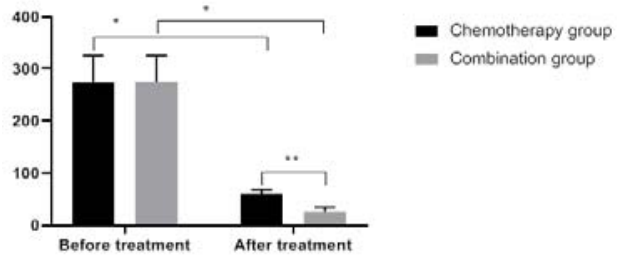

Figure 1: Comparison of HE4 levels. Values are expressed as mean \pm SD. ${ }^{*} P<0.001$, HE4 levels in chemotherapy group and combination group after treatment vs their levels before treatment; ${ }^{* *} p<0.001$, HE4 level in chemotherapy group after treatment vs HEA level in combination group after treatment

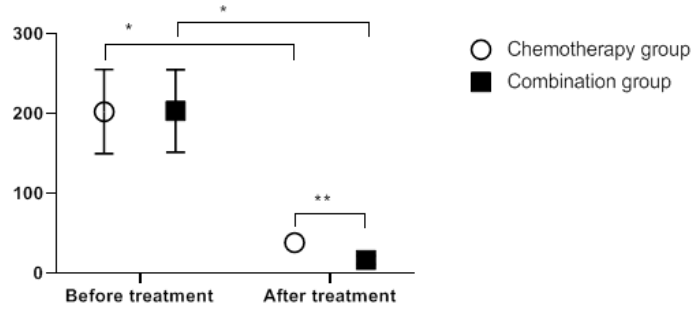

Figure 2: Comparison of CA125 levels. Data are expressed as mean \pm SD. ${ }^{*} P<0.001$, CA125 levels in the chemotherapy group and the combination group after treatment vs their levels before treatment; ${ }^{* *} p<$ 0.001 , CA125 level in chemotherapy group after treatment vs CA125 level in combination group after treatment

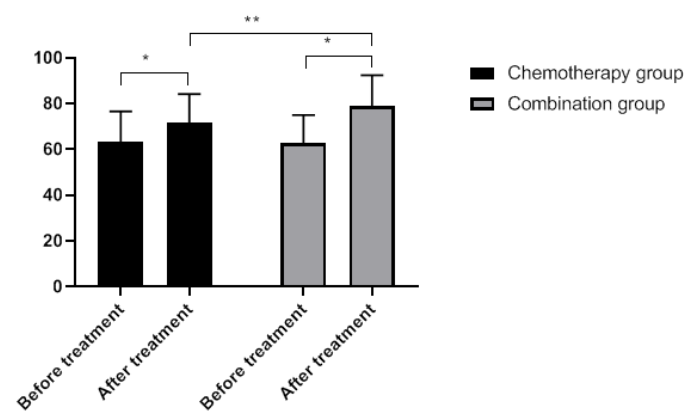

Figure 3: Comparison of KPS scores. Values are presented as mean $\pm \mathrm{SD}$. ${ }^{*} P<0.001$, KPS scores in the chemotherapy group and the combination group after treatment vs the corresponding scores before treatment; ${ }^{* *} p<0.001$, KPS score in the chemotherapy group after treatment vs KPS score in the combination group after treatment

\section{DISCUSSION}

Clinical research on recurrent ovarian cancer (ROC) has been gradually deepened in recent times. At present, the prognosis of ROC has been greatly improved, although many patients still suffer from varying degrees of recurrence within 1-2 years after treatment [13-16]. Medical research data show that secondary gene mutation is inevitable in ROC treatment: new gene mutations are the root causes of enhanced invasion and drug resistance of ROC. Indeed, patients who relapse within a short period of time have a high probability of carrying drug-resistant genes, leading to unsatisfactory inhibitory effect of chemotherapy on cancer. In recent years, great achievements have been made in clinical practice using TCM. Modern TCM has put forward the theories of blood stasis and toxins in summarizing the pathological features of various diseases. In a study by Wu et al [17], the etiology of ovarian diseases was attributed to blood stasis in the uterus and kidney yang deficiency, leading to entry of toxins and cold wind into the body. In addition, blood stasis in the body and deficiency of vital qi result in cold coagulation and $q i$ stagnation, all of which accumulate and produce a mass in the uterus. It has been revealed that ROC patients often show qi deficiency and blood stasis, while Wenyang Yiqi Jianpi Decoction dissolves blood stasis, detoxifies the blood and supports body resistance, with significant benefits for treatment of ROC [18].

In the prescription of Yiqi Jianpi Yangxue Decoction, Astragalus membranaceus plays the role of promoting yang diuresis, and tonifying middle-jiao and qi; while Curcuma zedoary removes blood stasis, dissolves lumps and relieves pain by promoting $q i$ circulation, thereby greatly enhancing blood stasis removal and alleviation of pain when it is combined with rhizoma sparganii. In addition, bighead atractylodes rhizome clears dampness, promotes diuresis, invigorates the spleen and supplements qi. Dangshen strengthens the spleen, nourishes the lung, and tonifies middle-Jiao and qi. Poria cocos strengthens the spleen, calms the heart, promotes diuresis and clears dampness. Kuhseng clears heat and dry dampness. Paeonia lactiflora nourishes the blood and regulates 
menstruation; it calms the liver and relieves pain. Oldenlandia clears heat and removes toxicity, while monkshood stops pain by eliminating cold; it tonifies fire and helps yang. Ginger stops cough by warming the lungs; it checks vomiting by warming the middle; it resolves the exterior and dissipates cold. The combined use of these drugs results in removal of blood stasis, detoxification, supplementation of qi and promotion of yang.

The present study has demonstrated that total treatment effectiveness was markedly higher in the combination group., and the HE4 and CA125 levels after treatment in the combination group were lower compared with the chemotherapy group. Serum human epididymis secretory protein 4 (HE4) protects the immune function, while CA125 is a tumor marker used for diagnosing ovarian cancer with high sensitivity, and it is highly expressed in the epithelial tissue of ovarian cancer. Thus, simultaneous assays of levels of HE4 and CA125 are beneficial in the diagnosis of ovarian cancer.

The levels of $\mathrm{CD}_{3}{ }^{+}, \mathrm{CD}_{4}{ }^{+}, \mathrm{CD}_{8}{ }^{+}$and NK cells in the combination group were higher, indicating enhanced the immune function of patients caused by combined therapy. After treatment, the KPS score in the combination group was higher. In TCM, it is believed that Astragalus membranaceus exerts anticancer effect by inhibiting tumor cell proliferation, promoting apoptosis and inhibiting angiogenesis. Astragalus membranaceus contains a large number of saponins, flavones and polysaccharides which can restore red blood cells, white blood cells and megakaryocytes in bone marrow, thereby reducing the inhibitory effect of chemotherapy on bone marrow. In addition, Kuh-seng exerts strong anti-tumor effect: it inhibits angiogenesis and prevents proliferation. Bighead atractylodes rhizome exerts anti-inflammatory effects, increases the level of white blood cells, improves the synthesis of small intestinal protein and regulates intestinal activity. Dangshen enhances immune function, protects the gastric mucosa of patients, and reduces gastrointestinal reactions caused by chemotherapy.

\section{Limitations of the study}

This study was a single-center study with small number of patients. In order to further confirm the effect of Yiqi Jianpi Yangxue Decoction on ROC patients, multi-center studies with more patients are needed.

\section{CONCLUSION}

This study has demonstrated that the combination of Yiqi Jianpi Yangxue Decoction and chemotherapy significantly enhanced clinical efficacy in the treatment of ROC. The combined treatment has high safety, and improves the health status and life quality of patients.

\section{DECLARATIONS}

\section{Conflict of Interest}

No conflict of interest associated with this work.

\section{Contribution of Authors}

We declare that this work was done by the authors named in this manuscript, and all liabilities pertaining to claims relating to the content of this manuscript will be borne by the authors. Qingling Zhang conceived and designed the study, and drafted the manuscript. Wenjuan Zhang and Xiao Wang collected, analyzed and interpreted the experimental data. Xueling Chen and Yewu Gao revised the manuscript for important intellectual content. All authors read and approved the final manuscript.

\section{Open Access}

This is an Open Access article that uses a funding model which does not charge readers or their institutions for access and distributed under the terms of the Creative Commons Attribution License (http://creativecommons.org/licenses/by/ 4.0) and the Budapest Open Access Initiative (http://www.budapestopenaccessinitiative.org/rea d), which permit unrestricted use, distribution, and reproduction in any medium, provided the original work is properly credited.

\section{REFERENCES}

1. Lin J, Li XL, Song $H$, Li Q, Wang MY, Qiu XM, Li DJ, Wang $L$. A general description for Chinese medicine in treating premature ovarian failure. Chin J Integr Med. 2017;23(2):91-97.

2. Friedlander ML. Do all patients with recurrent ovarian cancer need systemic therapy? Cancer. 2019;125 Suppl 24:4602-4608.

3. Sehouli J, Tomè O, Dimitrova $D$, Camara $O$, Runnebaum IB, Tessen HW, Rautenberg B, Chekerov R, Muallem $M Z$, Lux MP, et al. A phase III, open label, randomized multicenter controlled trial of oral versus intravenous treosulfan in heavily pretreated recurrent ovarian cancer: a study of the North-Eastern German Society of 
Gynecological Oncology (NOGGO). J Cancer Res Clin Oncol. 2017;143(3):541-550.

4. Chang JS, Kim SW, Kim YJ, Kim JY, Park SY, Kim JH, Jang TK, Kim YB. Involved-field radiation therapy for recurrent ovarian cancer: Results of a multi-institutional prospective phase II trial. Gynecol Oncol. 2018;151(1):39-45.

5. Günel T, Gumusoglu E, Dogan B, Ertem FB, Hosseini MK, Cevik N, Senol T, Topuz S, Aydinli K. Potential biomarker of circulating hsa-miR-1273g-3p level for detection of recurrent epithelial ovarian cancer. Arch Gynecol Obstet. 2018;298(6):1173-1180.

6. Jiang G, Balboni T, Taylor A, Liu J, Lee LJ. Palliative Radiation Therapy for Recurrent Ovarian Cancer: Efficacy and Predictors of Clinical Response. Int J Gynecol Cancer. 2018;28(1):43-50.

7. Bogani G, Leone Roberti Maggiore U, Chiappa V, Ditto A, Martinelli $F$, Sabatucci I, Mosca L, Lorusso $D$ Raspagliesi $F$. The addition of lymphadenectomy to secondary cytoreductive surgery in comparison with bulky node resection in patients with recurrent ovarian cancer. Int J Gynaecol Obstet. 2018;143(3):319-324.

8. Zhang Y, Mei Q, Liu Y, Li X, Brock MV, Chen M, Dong L, Shi L, Wang $Y$, Guo $M$, et al. The safety, efficacy, and treatment outcomes of a combination of low-dose decitabine treatment in patients with recurrent ovarian cancer. Oncoimmunology. 2017;6(9): e1323619.

9. PEBC's Ovarian Oncology Guidelines Group. A systematic review of patient values, preferences and expectations for the treatment of recurrent ovarian cancer. Gynecol Oncol. 2017;146(2):392-398.

10. World Medical Association. World Medical Association Declaration of Helsinki: ethical principles for medical research involving human subjects. JAMA. 2013;310(20):2191-4.

11. de Rooij BH, Ikiz H, Boll D, Pijnenborg JMA, Pijlman BM, Kruitwagen RFPM, van de Poll-Franse LV, Vos MC, Ezendam NPM. Recurrent Cancer Is Associated With
Dissatisfaction With Care-A Longitudinal Analysis Among Ovarian and Endometrial Cancer Patients. Int $J$ Gynecol Cancer. 2018;28(3):614-622.

12. Tung $C H$, Kuo LW, Huang MF, Wu YY, Tsai $Y T, W u J E$, Hsu KF, Chen YL, Hong TM. MicroRNA-150-5p promotes cell motility by inhibiting c-Myb-mediated Slug suppression and is a prognostic biomarker for recurrent ovarian cancer. Oncogene. 2020;39(4):862-876.

13. Tanyi JL, Stashwick C, Plesa G, Morgan MA, Porter D, Maus MV, June $\mathrm{CH}$. Possible Compartmental Cytokine Release Syndrome in a Patient With Recurrent Ovarian Cancer After Treatment With Mesothelin-targeted CART Cells. J Immunother. 2017;40(3):104-107.

14. Wang $X$, Han L, Zhou L, Wang L, Zhang LM. Prediction of candidate RNA signatures for recurrent ovarian cancer prognosis by the construction of an integrated competing endogenous RNA network. Oncol Rep. 2018;40(5):2659-2673.

15. Shoji $T$, Eto $H$, Sato $T$, Soma R, Fukagawa $D$, Tomabechi $H$, Takatori E, Nagasawa T, Sato S, Kagabu $M$ et al. A New Therapeutic Strategy for Recurrent Ovarian Cancer-Bevacizumab beyond Progressive Disease. Healthcare (Basel). 2019;7(3):109.

16. Morgan RD, Banerjee S, Hall M, Clamp AR, Zhou C, Hasan J, Orbegoso C, Taylor S, Tugwood J, Lyon AR, et al. Pazopanib and Fosbretabulin in recurrent ovarian cancer (PAZOFOS): A multi-centre, phase $1 \mathrm{~b}$ and openlabel, randomised phase 2 trial. Gynecol Oncol. 2020;156(3):545-551.

17. Wu L, Zhong L. Budget impact analysis of niraparib and olaparib for maintenance treatment of platinumsensitive, recurrent ovarian cancer in the US. J Med Econ. 2019;22(2):187-195.

18. Linus-Lojikip S, Subramaniam V, Lim WY, Hss AS. Survival of patients with advanced and recurrent ovarian cancer treated using integrative medicine in Malaysia: A case series. Complement Ther Clin Pract. 2019; 37:7385. 